\title{
Tasa de ovulación, prolificidad y tasa de gestación en cabras tratadas con glicerol por vía oral
}

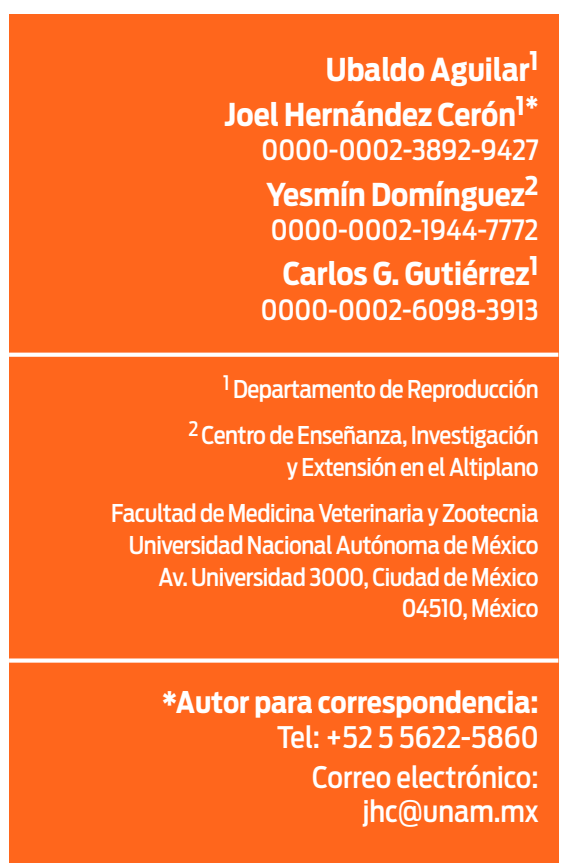

Recibido: 2015-10-12 Aceptado: 2016-03-16 Publicado: $2016-03-28$

Información y declaraciones adicionales en la página 8

(a) Derechos de autor: Ubaldo Aguilar et al. 2016

acceso abierto $\boldsymbol{\odot}$

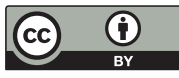

Distribuido bajo una Licencia Creative Commons Atribución 4.0 Internacional (CC-BY 4.0)

\section{Resumen}

Se probó si la administración oral de glicerol, al momento de retirar el progestágeno y durante los primeros seis días del ciclo estral, incrementaba la tasa de ovulación, la prolificidad y la tasa de gestación en cabras. Se insertaron esponjas intravaginales con acetato de fluorogestona a 129 cabras por 12 días; al retirar la esponja se asignaron al azar a uno de dos tratamientos: glicerol $(n=65)$ recibió $100 \mathrm{~mL}$ de glicerol por vía oral, mismo tratamiento que se repitió los días $0,2,4$ y 6 (estro = día 0), y testigo $(n=64)$, el cual no recibió glicerol. Las cabras en estro recibieron monta. Entre los días 8 y 12 del ciclo estral, se determinó la tasa de ovulación mediante ecografía. El diagnóstico de gestación se realizó mediante una ecografía el día 40 y la prolificidad se determinó al parto. En seis cabras tratadas con glicerol y cinco testigos, se determinaron las concentraciones de insulina mediante radioinmunoensayo a las $0,2,4,8$ y 12 h después de la ingestión de glicerol. La proporción de cabras con ovulación múltiple (glicerol $=71$ vs testigo $=64$ ) y la proporción de cabras con parto múltiple (glicerol $=52$ vs testigo $=56$ ) fueron similares ( $P>0.05)$ entre los tratamientos. De manera similar, la tasa de gestación fue igual $(P>0.05)$ entre los tratamientos (glicerol $=88$ vs testigo $=85 \%)$. Las concentraciones de insulina tendieron a ser mayores en las cabras tratadas con glicerol $(P=0.08)$. Se concluye que la administración oral de $100 \mathrm{~mL}$ de glicerol al momento de retirar el progestágeno y durante los primeros seis días del ciclo estral, no incrementó la tasa de ovulación, la prolificidad, ni la tasa de gestación en cabras.

Palabras clave: Tasa de ovulación; Prolificidad; Clicerol; Insulina; Cabras.

\section{Introducción}

El aumento de la tasa de ovulación y la prolificidad en pequeños rumiantes se ha logrado de manera práctica mediante tratamientos hormonales y por medio del incremento del aporte energético en la dieta (flushing). Aunque aún no se comprende completamente el mecanismo de acción del flushing, las evidencias indican que sus efectos se producen a nivel ovárico, son independientes de las concentraciones de gonadotropinas y se relacionan con un incremento en los ni- 
veles sanguíneos de glucosa e insulina (Downing et al., 1995; Muñoz-Gutiérrez et al., 2004) Dupont et al., 2014).

En la oveja, la suplementación durante un periodo corto con grano lupino una leguminosa con alto contenido de proteína y energía metabolizable- puede incrementar la tasa de ovulación. La suplementación con grano lupino estimula la folículogenesis e incrementa la tasa de ovulación, sin inducir cambios en el peso o en la condición corporal (Scaramuzzi et al., 2006). La glucosa y la insulina pueden influir en el aumento de la tasa de ovulación, ya que favorecen el desarrollo folicular y se relacionan con una mayor cantidad de folículos capaces de responder al estímulo de las gonadotropinas (Gutiérrez et al., 1997; Viñoles et al., 2005). Asimismo, la glucosa y la glucosamina estimulan la capacidad esteroidogénica de los folículos mediante un aumento en la actividad del factor de crecimiento parecido a la insulina tipo I (IGF-I; Muñoz-Gutiérrez et al., 2004). En estudios realizados en cabras, se observa que el aumento de insulina inducido por la dieta o por la administración de insulina incrementa la tasa de ovulación (Suguna et al., 2009; Zabuli et al., 2010).

El aumento de las concentraciones sanguíneas de glucosa e insulina puede lograrse administrando soluciones glucogénicas. En ovejas se han utilizado tratamientos cortos con glicerol y propilenglicol para aumentar la tasa de ovulación. Rodríguez-Iglesias et al. (1996) lograron aumentar la tasa de ovulación en ovejas anéstricas mediante la administración oral de $100 \mathrm{~mL}$ de una solución de glicerol y propilenglicol antes de la exposición a los machos. Gutiérrez et al. (2011) lograron incrementar las concentraciones séricas de glucosa, insulina y la tasa de ovulación con una sola toma de $300 \mathrm{~mL}$ de glicerol al momento de la inducción de la luteólisis con PGF2 $\alpha$ en ovejas Pelibuey. En este mismo estudio, lograron un efecto similar en la tasa de ovulación con una sola toma de $100 \mathrm{~mL}$ de glicerol.

Por otra parte, la insulina favorece el desarrollo embrionario temprano. En embriones bovinos producidos in vitro, la insulina ejerce un efecto mitogénico y antiapoptótico (Byrne et al., 2002; Augustin et al., 2003), lo que resulta en un aumento en la proporción de embriones que llegan a la etapa de blastocisto. Cabe señalar, también, que altas concentraciones de insulina tienen un efecto negativo en el desarrollo embrionario en el ganado bovino (Fouladi-Nashta et al., 2006) y ovino (Carrera-Chávez et al., 2014). Además, se ha observado un efecto positivo de la insulina sobre la función lútea. En un estudio realizado en cabras, la administración de insulina se relacionó con concentraciones más altas de progesterona durante la gestación (Suguna et al., 2009). Se conoce que niveles altos de progesterona se asocian con un desarrollo embrionario acelerado (Garret et al., 1988), lo cual podría reflejarse en menos pérdidas embrionarias. En vacas lecheras, la administración de $1 \mathrm{~L}$ de glicerol durante los primeros seis días posteriores a la inseminación, incrementa las concentraciones sanguíneas de insulina y favorece la tasa de gestación (Ortega et al., 2010). Por lo tanto, en el presente trabajo se probó si la administración oral de $100 \mathrm{~mL}$ de glicerol al momento de retirar el progestágeno y durante los primeros seis días del ciclo estral, incrementaba la tasa de ovulación, la prolificidad y la tasa de gestación en cabras. 


\section{Materiales y métodos} Localización

El experimento se llevó a cabo de septiembre a noviembre -periodo comprendido dentro de la época reproductiva de las cabras en México (Arvizu et al., 1995) - en un centro experimental de la Facultad de Medicina Veterinaria y Zootecnia de la UNAM, localizado en Tequisquiapan, Querétaro (latitud: $20^{\circ} 31^{\prime} 21^{\prime \prime} \mathrm{N}$ y altitud: $1881 \mathrm{msnm}$ ). El clima de la región es semiseco templado, con una temperatura anual promedio de $17.5^{\circ} \mathrm{C}$ y una precipitación pluvial media anual de $511.8 \mathrm{~mm}$ (García, 1981). .

\section{Animales}

Se utilizaron 129 cabras (22 primalas y 107 multíparas) de raza cruzada (BoerAlpino Francés), ciclando y con condición corporal entre 2 y 3 (Russel et al., 1969). Las cabras permanecieron en estabulación y se alimentaron con una ración basada en heno de alfalfa, ensilado de maíz y concentrado, lo cual cubría sus necesidades de acuerdo con las necesidades marcadas en los cuadros del National Research Council (NRC). El manejo de los animales durante el experimento fue aprobado por el Consejo Académico del Posgrado en Ciencias de la Producción y de la Salud Animal de la Universidad Nacional Autónoma de México.

\section{Tratamientos}

A todas las cabras se les insertó una esponja intravaginal impregnada con $45 \mathrm{mg}$ de acetato de fluorogestona (FGA; Chronogest; MSD Salud Animal, México; Intervet México, S.A. de C.V., Huixquilucan, Estado de México, México), la cual permaneció in situ durante 12 días. Al retirar la esponja se aplicó una dosis luteolítica de cloprostenol sódico (Celosil; MSD Salud Animal, México) y las cabras se asignaron al azar a dos tratamientos: en el grupo de glicerol $(n=65)$, los animales recibieron por vía oral $100 \mathrm{~mL}$ de una solución a base de glicerol y agua (90:10 v/v), la cual se repitió los días $0,2,4$ y 6 post-estro (estro = día 0 ), y las cabras en el grupo testigo $(n=64)$ no recibieron glicerol. La detección de estros se realizó con machos provistos de un mandil. Las hembras detectadas en estro recibieron una monta con machos de fertilidad probada.

Entre los días 8 y 12 posmonta, se determinó la tasa de ovulación mediante ecografía transrectal contando el número cuerpos lúteos; para este propósito se utilizó un transductor lineal de $7.5 \mathrm{MHz}$ (Aloka, Co., LTD, Tokyo, Japan), al cual se le adaptó un soporte rígido que permitió su manipulación. La tasa de gestación se determinó mediante el diagnóstico de gestación por ecografía el día 40 después del servicio. La determinación de la prolificidad se llevó a cabo al momento del parto (figura 1).

\section{Muestreo sanguíneo y procesamiento de las muestras}

Para determinar las concentraciones sanguíneas de progesterona e insulina, se muestreó la sangre de seis cabras tratadas con glicerol y la de cinco testigos seleccionadas al azar. Las muestras para cuantificar las concentraciones sanguíneas de progesterona se tomaron diariamente entre los días 1 y 17 del ciclo estral. Los ciclos de dos cabras del grupo testigo fueron cortos (regresión prematura del 

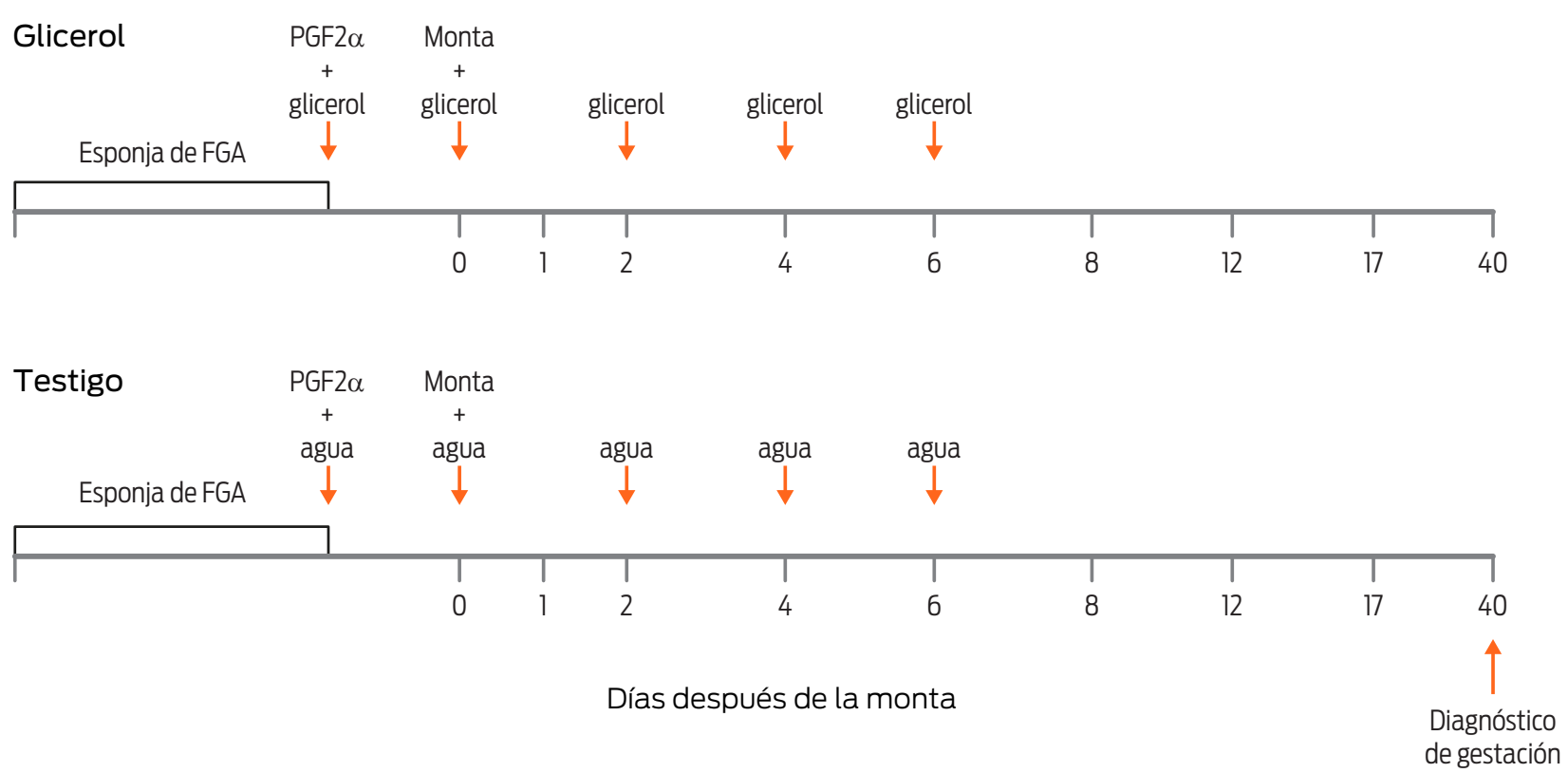

Figura 1. Esquema de los tratamientos.

cuerpo lúteo), por lo que estas cabras no se incluyeron en el análisis estadístico de progesterona. Se realizó un muestreo el día 4 del ciclo estral para determinar las concentraciones sanguíneas de insulina inducidas por la administración oral de glicerol. La primera muestra se tomó antes del tratamiento $(0 \mathrm{~h})$ y las siguientes a las $2,4,8$ y 12 h después de la ingesta del glicerol.

Las muestras de sangre $(10 \mathrm{~mL})$ se obtuvieron por punción de la vena yugular, en tubos Vacutainer ${ }^{\circledR}$ adicionados con $100 \mu \mathrm{L}$ de citrato de sodio. Las muestras se centrifugaron a $1500 \mathrm{xg}$ durante 10 minutos para separar el plasma, el cual se conservó a $-20{ }^{\circ} \mathrm{C}$ hasta su análisis. Las concentraciones de progesterona e insulina se determinaron mediante radioinmunoanálisis en fase sólida (Coat-A-Count ${ }^{\mathbb{R}}$, RIA kit, DPC; USA). En el caso de progesterona, la sensibilidad del ensayo fue de $0.1 \mathrm{ng} / \mathrm{mL}$, con un coeficiente de variación intraensayo de $4.2 \%$, mientras que para insulina la sensibilidad fue $0.052 \mathrm{ng} / \mathrm{mL}$, con un coeficiente de variación intraensayo de $3.3 \%$.

\section{Análisis estadístico}

Se utilizó un modelo de regresión logística para determinar el efecto del tratamiento con glicerol en la proporción de cabras con ovulación y partos múltiples. Asimismo, mediante regresión logística, se analizó el efecto del número de partos (primíparas y multíparas) y su interacción con el tratamiento [SAS version 9.2 (SAS Institute Inc., Cary, NC)]. La tasa de ovulación y la prolificidad se compararon mediante la prueba $U$ de Mann-Whitney, en la cual se consideró el efecto del tratamiento y su interacción con el número de partos. Las concentraciones de progesterona e insulina se compararon por medio de un análisis de varianza para mediciones repetidas; el modelo incluyó el tratamiento, el tiempo y su interacción. En todos los casos se consideró como diferencia estadística con un valor de $\mathrm{P}<0.05$ y una tendencia con valor de $\mathrm{P} \leq 0.10$. 


\section{Resultados y discusión}

La administración oral de $100 \mathrm{~mL}$ de glicerol al momento de retirar el progestágeno no incrementó la tasa de ovulación; la proporción de cabras con ovulación múltiple y partos múltiples fue similar entre los tratamientos (cuadros 1 y 2). La tasa de ovulación [glicerol $=1.84 \pm 0.64$ vs testigo $=1.80 \pm 0.72$ (media \pm desviación estándar)] y la prolificidad [glicerol $=1.58 \pm 0.61$ vs testigo $=1.60 \pm 0.64$ (media \pm desviación estándar)] fueron similares entre los tratamientos. Estos resultados son diferentes a los encontrados en ovejas por Rodríguez Iglesias et al. (1996) y Gutiérrez et al. (2011). La causa por la cual el tratamiento no tuvo un efecto favorable en la tasa de ovulación puede estar relacionada con la respuesta metabólica a la dosis de glicerol administrada. La dosis de $100 \mathrm{~mL}$ de glicerol se determinó con base en los resultados obtenidos en la oveja (Gutiérrez et al. 2011); en dicho estudio, la administración de $100 \mathrm{~mL}$ de glicerol provocó un incremento de la tasa de ovulación similar al obtenido con $300 \mathrm{~mL}$, por lo cual se decidió administrar $100 \mathrm{~mL}$.

En esta investigación, las concentraciones séricas de insulina no se vieron afectadas por el tratamiento $(P=0.11)$, pero sí hubo una interacción entre el tratamiento y el tiempo $(P=0.08)$. Así, las concentraciones de insulina tendieron a ser mayores en las siguientes 2 y 4 horas postratamiento (figura 2). Posiblemente este aumento discreto de las concentraciones de insulina no fue suficiente para favorecer la tasa de ovulación.

La causa de la variación en la respuesta a la misma dosis de glicerol observada entre la oveja y la cabra se desconoce. Se han evaluado soluciones glucogénicas en ovejas y vacas, pero no en cabras (Rodríguez-Iglesias et al. 1996; Ortega et al., 2010; Gutiérrez et al. 2011); aunque estas tres especies son rumiantes, tienen diferencias en su función digestiva y en el aprovechamiento de los forrajes. Las cabras poseen adaptaciones fisiológicas que les permiten aprovechar de manera más eficiente los alimentos con alto contenido de fibra (Silanikove, 2000), por lo cual pueden ser menos sensibles a los cambios en la calidad y cantidad del alimento ofrecido. El glicerol por vía oral se utiliza como un sustrato en la fermentación ruminal, a partir del cual se puede obtener propionato o también se puede absorber directamente como glicerol a través de la mucosa ruminal, que después se convertirá en glucosa en el hígado (Trabue et al., 2007). Aunque es la misma ruta metabólica en los rumiantes, su eficiencia para desencadenar la secreción de insulina puede ser diferente entre especies; esto explicaría, en parte, el éxito del tratamiento para aumentar la tasa de ovulación en la oveja y el fracaso del mismo tratamiento en la cabra. Los resultados del presente trabajo permiten diseñar estudios futuros para la cabra con el fin de evaluar diferentes dosis de glicerol hasta encontrar la dosis que desencadene una respuesta óptima en las concentraciones séricas de insulina.

En este análisis se propuso que la administración oral de glicerol durante los primeros días del desarrollo del embrión (días 0, 2, 4 y 6 del ciclo estral) favorecería la sobrevivencia embrionaria, lo cual incrementaría la proporción de cabras gestantes y la prolificidad. Esta hipótesis se sustentó en el efecto esperado del glicerol en las concentraciones de insulina y en los efectos que tiene la insulina en el desarrollo embrionario. En estudios realizados in vitro e in vivo se observa que la insulina mejora el desarrollo embrionario mediante un aumento de la proliferación celular y la disminución de la apoptosis (Augustin et al., 2003; Suguna et al., 2009), mientras que concentraciones bajas de insulina afectan negativamente el desarrollo embrionario y su capacidad para producir interferón tau (Thatcher et al., 
Cuadro 1. Porcentaje de cabras con ovulación múltiple tratadas con 100 mL de glicerol por vía oral.

\begin{tabular}{|c|c|c|c|c|c|}
\hline Variable & Clase & $\begin{array}{c}\text { Porcentaje } \\
\text { de ovulaciones múltiples }\end{array}$ & $\begin{array}{c}\text { Razón } \\
\text { de momios }\end{array}$ & $\begin{array}{c}\text { Intervalo } \\
\text { de confianza (95\%) }\end{array}$ & Valor de P \\
\hline \multirow{2}{*}{ Tratamiento } & Testigo & $\begin{array}{c}64 \\
(29 / 45)\end{array}$ & 1.00 & & 0.260 \\
\hline & Glicerol & $\begin{array}{c}71 \\
(35 / 49)\end{array}$ & 1.73 & $0.66-4.49$ & \\
\hline \multirow{2}{*}{$\begin{array}{c}\text { Número } \\
\text { de partos }\end{array}$} & Primíparas & $\begin{array}{c}31 \\
(5 / 16)\end{array}$ & 1.00 & & 0.001 \\
\hline
\end{tabular}

Cuadro 2. Porcentaje de cabras con parto múltiple tratadas con $100 \mathrm{~mL}$ de glicerol por vía oral.

\begin{tabular}{|c|c|c|c|c|c|}
\hline Variable & Clase & $\begin{array}{c}\text { Porcentaje } \\
\text { de partos múltiples }\end{array}$ & $\begin{array}{c}\text { Razón } \\
\text { de momios }\end{array}$ & $\begin{array}{c}\text { Intervalo } \\
\text { de confianza (95\%) }\end{array}$ & Valor de P \\
\hline \multirow{2}{*}{ Tratamiento } & Testigo & $\begin{array}{c}56 \\
(26 / 46)\end{array}$ & 1.00 & & \\
& Clicerol & $\begin{array}{c}52 \\
(25 / 48)\end{array}$ & 0.95 & $0.40-2.20$ & 0.906 \\
\hline \multirow{2}{*}{$\begin{array}{c}\text { Número } \\
\text { de partos }\end{array}$} & Primíparas & $\begin{array}{c}27 \\
(4 / 15)\end{array}$ & 1.00 & & 0.002 \\
\hline
\end{tabular}

1995). Además, en vacas lecheras, un incremento en las concentraciones de insulina inducido por la administración de un litro de glicerol por vía oral en los primeros seis días del desarrollo embrionario (días $0,2,4$, y 6 ) incrementó la tasa de gestación (Ortega et al., 2010). Sin embargo, en esta investigación, la tasa de gestación (glicerol $=88$ vs testigo $=85 \%$ ), la proporción de partos múltiples y la prolificidad fueron similares entre los tratamientos ( $P>0.05$; cuadro 2). La carencia de efecto en la prolificidad y en la tasa de gestación, aquí observadas, probablemente obedece al incremento marginal de las concentraciones de insulina después de la ingesta de $100 \mathrm{~mL}$ de glicerol. Además, la tasa de gestación obtenida en ambos grupos fue alta, lo que hace más difícil ver los efectos favorables de cualquier tratamiento dirigido a mejorar la fertilidad. El número de partos afectó la tasa de ovulación y la prolificidad, pero esta variable no interactúo con el tratamiento (cuadros 1 y 2 ; $P>0.1)$.

También se propuso que la administración de glicerol durante los primeros días del ciclo estral podría favorecer el desarrollo embrionario y la tasa de gestación mediante el incremento de las concentraciones séricas de progesterona. Lo anterior se fundamenta en estudios en bovinos, en los cuales la insulina incrementa las concentraciones circulantes de progesterona (Spicer et al., 1995; Cooke et al., 2012). En este estudio, sin embargo, las concentraciones de progesterona entre los días 1 y 17 después de la monta fueron similares entre los grupos y no se observó interacción entre el tratamiento y el tiempo ( $P>0.05$; figura 3).

La buena condición corporal de las cabras, así como la estación del año en la cual se hizo el experimento, quizá influyeron en la ausencia de un efecto positivo del glicerol. Las cabras recibieron una dieta que cubría los requerimientos señala- 


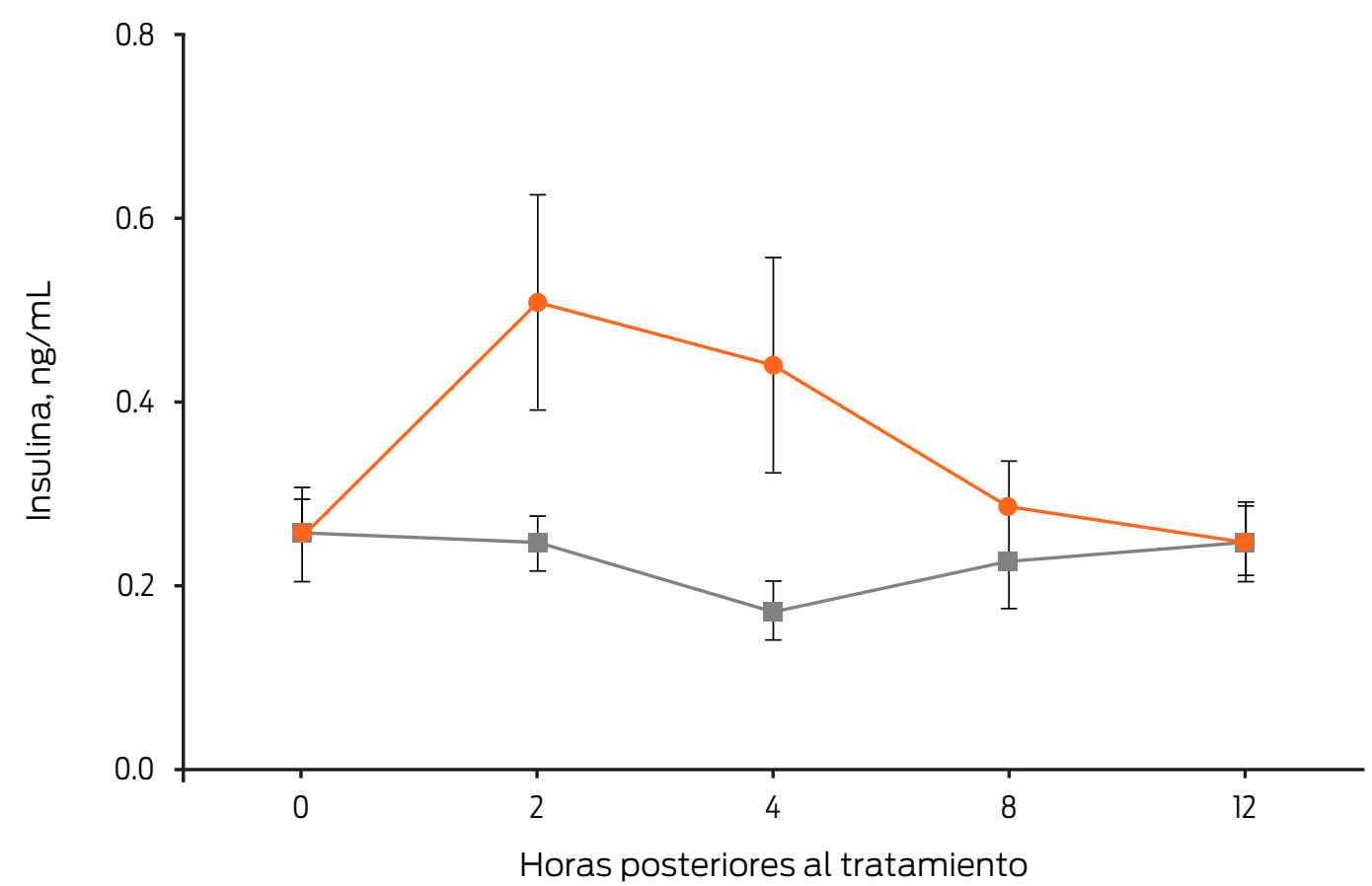

Figura 2. Concentraciones promedio de insulina ( \pm error estándar) en cabras tratadas con $100 \mathrm{~mL}^{-1}$ de glicerol $(-\bullet-)$ y testigos ( - -). Interacción tratamiento por tiempo: $\mathrm{P}=0.08)$.

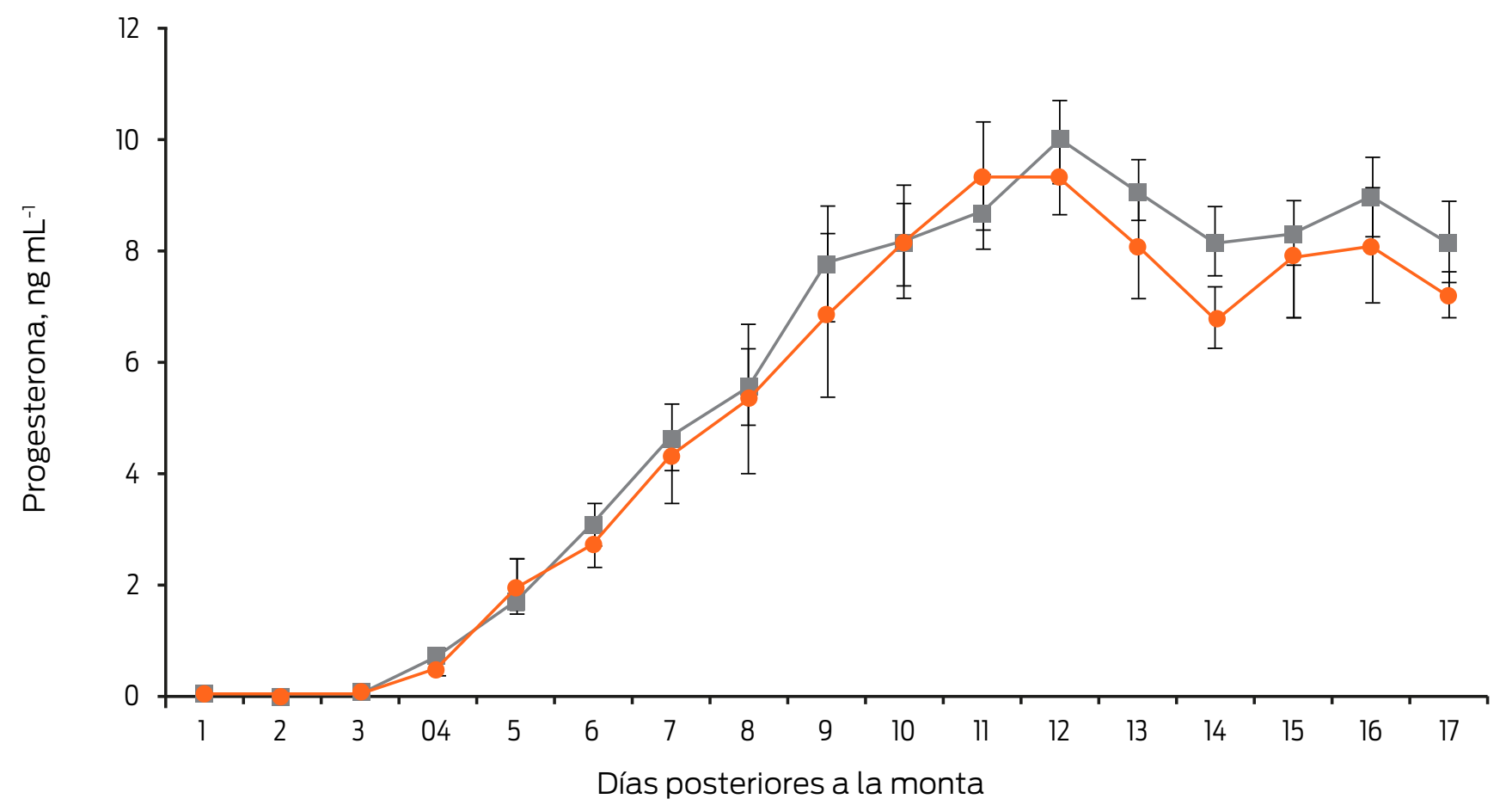

Figura 3. Concentraciones promedio de progesterona ( \pm error estándar) en cabras tratadas con $100 \mathrm{~mL}$ de glicerol $(\rightarrow-)$ y cabras testigo $(-\rightarrow)(P>0.05)$. 
dos en el NRC y su condición corporal estaba entre 2 y 3 al inicio del experimento, lo que reflejó una tasa de gestación y prolificidad altas, similares a las observadas consistentemente en el rebaño en el que se hizo el trabajo. Por otro lado, este estudio se hizo durante plena época reproductiva, factor que también contribuyó con la excelente respuesta en fertilidad. Por ello, se considera que los experimentos subsiguientes se deberán hacer en condiciones en las cuales la tasa de gestación y la prolificidad estén disminuidas, como en los programas de inducción de la ciclicidad durante la estación no reproductiva o en cabras con baja condición corporal. En estas condiciones la suplementación propuesta podría mejorar la fertilidad.

Existen tratamientos que no incrementan la tasa de gestación global, pero sí lo hacen en subgrupos de animales; en un estudio de nuestro grupo de investigación, por ejemplo, se observó que la somatotropina bovina recombinante incrementó la tasa de gestación en cabras anéstricas y la prolificidad en cabras primíparas, subgrupos de cabras que de manera natural tienden a tener una baja tasa de gestación y una baja prolificidad (Hernández y Gutiérrez, 2013).

\section{Conclusiones}

Se concluye que la administración oral de $100 \mathrm{~mL}$ de glicerol al momento de retirar el progestágeno y durante los primeros seis días del ciclo estral no incrementó la tasa de ovulación, la prolificidad ni la tasa de gestación en cabras.

\section{Financiamiento}

Parte de este estudio se financió con el proyecto PAPIIT IN22235 de la UNAM.

\section{Agradecimientos}

Los autores agradecen el apoyo generoso de los trabajadores y técnicos del Centro de Enseñanza, Investigación y Extensión en Producción Animal en Altiplano (CEIEPAA), Tequisquiapan, Querétaro.

\section{Conflictos de interés}

Los autores declaran que no hay conflictos de interés.

\section{Contribución de los autores}

Ubaldo Aguilar realizó el experimento y analizó los resultados.

Yesmín Domínguez realizó el experimento.

Joel Hernández Cerón y Carlos Gutiérrez diseñaron el experimento, analizaron los resultados y escribieron el manuscrito. 


\section{Referencias}

1) Arvizu R, Hernández Cerón J, Alberti A, Porras A, Valencia J. 1995. Inicio de la actividad ovárica posparto y características de la primera ovulación de cabras criollas paridas en dos épocas del año. Avances en Investigación Agropecuaria 4:9-15.

2) Augustin R, Pocar P, Wrenzycki C, Niemann H, Fischer B. 2003. Mitogenic and anti-apoptotic activity of insulin on bovine embryos produced in vitro. Reproduction 126:91-99.

3) Byrne AT, Southgate J, Brison DR, Leese HJ. 2002. Regulation of apoptosis in the bovine blastocyst by insulin and insulin-like growth factor (IGF) superfamily. Molecular Reproduction and Development 62:489-495.

4) Carrera-Chávez JM, Hernández-Cerón J, López-Carlos MA, Lozano-Domínguez RR, Molinar F, Echavarría-Cháirez FG, Bañuelos-Valenzuela R, Aréchiga CF. 2014. Superovulatory response and embryo development in ewes treated with two doses of bovine somatotropin. Animal Reproduction Science 151: 105-111.

5) Cooke RF, Cappellozza BI, Reis MM, Bohnert DW, Vasconcelos JL. 2012. Plasma progesterone concentration in beef heifers receiving exogenous glucose, insulin, or bovine somatotropin. Journal of Animal Science 90:3266-3273.

6) Downing JA, Joss J, Scaramuzzi RJ. 1995. Ovulation rate and the concentrations of gonadotrophins and metabolic hormones in ewes infused with glucose during the late luteal phase of the oestrous cycle. Journal of Endocrinology 146:403-410.

7) Dupont J, Scaramuzzi RJ, Reverchon M. 2014. The effect of nutrition and metabolic status on the development of follicles, oocytes and embryos in ruminants. Animal 8:1031-1044.

8) Fouladi-Nashta AA, Campbell KHS. 2006. Dissociation of oocyte nuclear and cytoplasmic maturation by the addition of insulin in cultured bovine antral follicles. Reproduction 131; 449-460.

9) García E. 1981. Modificaciones al Sistema de Clasificación Climática de Köppen. $5^{\text {a }}$ edición. México, D. F., México: Instituto de Geografía, UNAM.

10) Garret JE, Geisert RD, Zavy MT, Morgan GL. 1988. Evidence for maternal regulation of early conceptus growth and development in beef cattle. Journal of Reproduction and Fertility 84:437-446.

11) Gutiérrez CG, Oldham J, Bramley TA, Gong JG, Campbell BK, Webb R. 1997. The recruitment of ovarian follicles is enhanced by increased dietary intake in heifers. Journal of Animal Science 75:1876-1884.

12) Gutierrez CG, Ferraro $S$, Martinez V, Saharrea A, Cortez C, Lassala A, Basurto $H$, Hernandez Ceron J. 2011. Increasing ovulation quota: more than a matter of energy. Acta Scientiae Veterinariae 39 (Suppl 1):305-316.

13) Hernández-Cerón J, Gutiérrez CG. La somatotropina bovina recombinante y la reproducción en bovinos, ovinos y caprinos. Agrociencia 47: 35-45. 2013.

14) Muñoz-Gutiérrez M, Blache D, Martin GB, Scaramuzzi RJ. 2004. Ovarian follicular expression of mRNA encoding the type I IGF receptor and IGF-binding protein-2 in sheep following five days of nutritional supplementation with glucose, glucosamine or lupins. Reproduction 128:747-756.

15) Ortega LA, Hernández-Cerón J, Gutiérrez CG. 2010. La administración oral de glicerol después de la inseminación incrementa el porcentaje de concepción en vacas Holstein. Técnica Pecuaria en México 48:69-74 
16) Rodríguez-Iglesias RM, Ciccioli NH, Irazoqui $\mathrm{H}$, Giglioli C. 1996. Ovulation rate in ewes after single oral glucogenic dosage during a ram-induced follicular phase. Animal Reproduction Science 44:211-221.

17) Russel AJF, Doney JM, Gunn RG. 1969. Subjective assessment of body fat in live sheep. Journal of Agricultural Science 72:451-454.

18) Scaramuzzi RJ, Campbell BK, Downing JA, Kendall NR, Khalid M, Muñoz-Gutiérrez M, Somchit A. 2006. A review of the effects of supplementary nutrition in the ewe on the concentrations of reproductive and metabolic hormones and the mechanisms that regulate folliculogenesis and ovulation rate. Reproduction Nutrition Development 46:339-354.

19) Silanikove N. 2000. The physiological basis of adaptation in goats to harsh environments. Small Ruminant Research 35:181-193.

20) Spicer, LJ, Echternkamp SE. 1995. The ovarian insulin andinsulin-like growth factor system with an emphasis on domestic animals. Domestic Animal Endocrinology 12:223-245.

21) Suguna K, Mehrotra $S$, Agarwal SK, Hoque M, Shanker U, Singh SK, Varshney VP. 2009. Effect of exogenous insulin administration on ovarian function, embryo/ fetal development during pregnancy in goats. Animal Reproduction Science $111: 202-213$.

22) Thatcher WW, Meyer MD, Danet-Desnoyers G. 1995. Maternal recognition of pregnancy. Journal of reproduction and Fertility 49 (Suppl.), 15-28.

23) Trabue S, Scoggin K, Tjandrakusuma S, Rasmussen MA, Reilly PJ. 2007. Ruminal Fermentation of Propylene Glycol and Glycerol Journal of Agricultural and Food Chemistry 55:7043-7051

24) Viñoles C, Forsberg M, Martin GB, Cajarville C, Repetto J, Meikle A. 2005. Shortterm nutritional supplementation of ewes in low body condition affects follicle development due to an increase in glucose and metabolic hormones. Reproduction 129:299-309.

25) Zabuli J, Tanaka T, Lu W, Kamomae H. 2010. Intermittent nutritional stimulus by short-term treatment of high-energy diet promotes ovarian performance together with increases in blood levels of glucose and insulin in cyclic goats. Animal Reproduction Science 122:288-293. 